\title{
Transtorno de déficit de atençáo e hiperatividade: conhecendo para intervir
}

\author{
Attention deficit hyperactivity disorder: known for intervene
}

Sandro Cezar Moreira ${ }^{1}$

Maria Auxiliadora Motta Barreto ${ }^{2}$

\section{Resumo}

O artigo apresenta uma revisão bibliográfica sobre Transtorno de Déficit de Atenção e Hiperatividade (TDAH), reunindo alguns dos principais autores sobre o assunto. $\mathrm{O}$ objetivo é levantar informaçôes relevantes sobre o conceito do TDAH, como se diagnostica a doença, possíveis causas, prevalência, principais comorbidades e a influência negativa que o transtorno traz no desempenho de crianças em idade escolar. O TDAH é uma doença caracterizada por desatenção, hiperatividade e impulsividade e está presente em $5 \%$ das crianças no mundo. O diagnóstico é essencialmente clínico e cerca de 30\% a 50\% dos casos apresentam comorbidades, o que dificulta a avaliação, assim como o tratamento. É fundamental que profissionais da educação, como, pedagogos e professores tenham cada vez mais informaçôes sobre o TDAH, pois quanto maior o conhecimento, melhores poderão ser as estratégias para encaminhamento destas crianças para tratamento adequado e para minimizar seus problemas de aprendizagem.

Palavras-chave: Transtorno de Déficit de Atenção e Hiperatividade, Dificuldade de Aprendizagem, Educaçáo Inclusiva.

\section{Abstract}

The article presents a literature review on the Attention Deficit Hyperactivity Disorder (ADHD), bringing together some of the main authors on the subject. The goal is raising information about the concept of ADHD, as diagnosed the disease, possible causes, prevalence, comorbidities and major negative impact that the disorder has on the performance of school-age children. The ADHD is a disorder characterized by inattention, hyperactivity and impulsivity and it is presented in 5\% of children in the world. The diagnosis is mainly clinical and around 30\% to 50\% of cases present comorbidities, which complicates the evaluation and treatment. It is essential that professionals in education, as educators and teachers have more information about ADHD, because the greater the knowledge, may be the best routing strategies for these children to appropriate treatment and to minimize the problems of learning.

Keywords: Attention Deficit Hyperactivity Disorder, Learning Disabilities, Inclusive Education

\footnotetext{
${ }^{1}$ Discente do Programa de Mestrado em Ensino em Ciência da Saúde e do Meio Ambiente - UniFOA; Docente do Centro Universitário de Barra Mansa - UBM ; Especialista em Treinamento Esportivo em Alto Nível - UFRRJ; Licenciado em Educação Física - UniFOA ${ }^{2}$ Docente do Programa de Mestrado em Ensino em Ciência da Saúde e do Meio Ambiente - UniFOA ${ }^{2}$ Doutora em Psicologia como Profissão e Ciência, Mestre em Educação, Psicóloga.
} 


\section{INTRODUÇÃO}

O artigo propõe uma revisão bibliográfica sobre Transtorno de Déficit de Atenção e Hiperatividade (TDAH), uma vez que se percebe significativo aumento de casos diagnosticados de alunos com TDAH nas escolas de ensino fundamental.

A relevância de um estudo como este está no grande número de crianças e adolescentes portadoras do TDAH nas escolas, onde a prevalência média mundial gira em torno de 5\%, e na persistência na vida adulta de 60\% dos casos (GOMES et al, 2007). Alguns estudos como o de Fontana et al (2007) apresentam que, no Brasil, a prevalência é de 13\%. Quanto mais precoce o diagnóstico, menor o impacto negativo que o TDAH traz para a vida das crianças, logo um maior conhecimento dos professores sobre o assunto pode ajudar tanto no diagnóstico como no tratamento destas crianças.

Segundo Gomes et al (2007), no Brasil, é insuficiente o conhecimento acerca do TDAH, tanto na população em geral quanto em profissionais envolvidos no seu diagnóstico e tratamento. Como profissionais da educação, consideramos, então, imprescindíveis informaçôes consistentes e objetivas acerca do transtorno.

Nosso objetivo é reunir informaçôes relevantes sobre o tema, em particular: conceito, tipos existentes e suas principais características, prevalência, possíveis causas, como se diagnostica a doença, principais comorbidades e a influência negativa que o transtorno traz no desempenho das crianças em idade escolar.

\section{DESENVOLVIMENTO}

\section{Conceituaçáo}

O Transtorno de Déficit de Atenção e Hiperatividade (TDAH) se constitui numa patologia reconhecida pela Organização Mundial da Saúde. Segundo Cypel (2007) o conceito de TDAH é bastante genérico, diagnosticado puramente por critério clínico e marcado pela descrição de um conjunto de sinais e sintomas. Em geral, baseia-se na avaliação de manifestaçôes relacionadas à desatenção, à hiperatividade e à impulsividade.

Alguns autores, como Barkley (apud BENCZIK, 2000) acreditam que os sintomas do TDAH podem aparecer em crianças até os 12 anos, sendo definido pelo DSMIV (Diagnostic and Statistical Manual of Mental Disorders
- Fourth Edition) como um problema de saúde mental, considerado como um distúrbio bidimensional que envolve a atenção e a hiperatividade/impulsividade.

Para Rohde et al (2003), o TDAH é considerado um transtorno do desenvolvimento e já foi entendido como um distúrbio comportamental de meninos, porém, hoje é frequentemente diagnosticado em meninas, adolescentes e adultos. Segundo o autor o transtorno pode ser subdividido em três tipos, de acordo com a predominância dos sintomas: predominantemente desatento, predominantemente hiperativo/impulsivo e combinado.

Fontana et al (2007) e Benczik (2000) consideram que a característica fundamental do transtorno é um padrão persistente de desatenção e/ou hiperatividade, mais freqüente e intenso que aquele apresentado por indivíduos de nível equivalente de desenvolvimento.

Segundo Phelan (2005) existem oito características que normalmente acompanham o TDAH: a desatenção ou tendência à distração, a impulsividade, a dificuldade de esperar ser atendido, a hiperatividade, a super excitação emocional, a desobediência, os problemas sociais e a desorganização. A criança pode ter todas ou apenas algumas dessas características.

\section{Prevalência}

Ciasca (2003) e Benczik (2000) estimam que a prevalência do TDAH entre as crianças em idade escolar nos Estados Unidos esteja na ordem de 3\% a 5\%. Para Arnald e Jensen (apud ROHDE, 2003) a taxa de prevalência varia nos diferentes países: 3\% a 6\% na Nova Zelândia; 2\% a 6,7\% na Alemanha; $8,7 \%$ no Japão; $8,9 \%$ na China; $1 \%$ na Inglaterra e $4 \%$ na Itália ${ }^{3}$.

De acordo com Rohde (2003), vários estudos como os de Brow (2001); Szatmari (1989) e Brito (1995), apontam a preponderância do sexo masculino sobre o feminino, mas nenhuma explicação clara surgiu de qualquer área que confirme essa diferença. Já em outros estudos, como os de Goldetein (1998); Carlson et al (1997); Faraone et al (2000), essa preponderância não aparece, fazendo crer que a diferença está nas amostras, por serem encaminhados mais meninos do que meninas para tratamento (ROHDE, 2003).

No Brasil foram relatadas algumas prevalências do TDAH. Guardiola (apud BENCZIK, 2000) por meio de critérios neuropsicológicos, encontrou de 3,5\% a 3,9\% em uma amostra representativa de alunos da $1^{\circ}$ série na cidade de Porto Alegre. Outra pesquisa realizada por Rohde em 1999, com 1013 alunos de 12 a 14 anos por meio de crité-

\footnotetext{
${ }^{3}$ De acordo com o autor essa diferenciação é resultado das diferentes metodologias aplicadas
} 
rios de DSM-IV, apontou 5,8\% (ROHDE, 2003). E ainda Barbosa (apud BENCZIK, 2000) na Paraíba, por meio da escala de Canners, encontrou 3,3\%. Fontana et al (2007) em amostra de 461 alunos em 4 escolas de São Gonçalo, utilizando critérios de DSM-IV, encontrou 13\%.

Desta forma, a prevalência no Brasil varia muito de uma pesquisa para outra, mostrando a necessidade de uma uniformização melhor nos critérios diagnósticos e a necessidade de continuação dos estudos.

\section{Etiologia}

Apesar de todos os estudos realizados para tentar descobrir as possíveis causas do TDAH, elas ainda continuam desconhecidas e, embora hipóteses existam em abundância, não respondem satisfatoriamente por todos os casos. Nenhuma hipótese isolada obteve aceitação como causa, porém, várias foram apoiadas por evidências convincentes, focalizadas em alguma anormalidade de funcionamento cerebral, genética ou adquirida e até mesmo de socialização. (ARNOLD; JENSEN apud BARBOSA et al, 2005).

Segundo Benczik (2000) estudos em pessoas que tiveram traumatismo, tumores ou doenças na região frontal e começaram a apresentar sintomas parecidos com o TDAH apontam o comprometimento dessa área específica do cérebro. A região frontal é uma das mais desenvolvidas nos seres humanos e parece ser responsável pela inibição comportamental, pela capacidade de prestar atenção, pelo autocontrole e pelo planejamento para o futuro. O que parece estar alterado na região é o funcionamento dos sistemas de substâncias químicas, chamados neurotransmissores, principalmente a dopamina e a noradrenalina. Contudo, a idéia de que o TDAH seja causado por este motivo ainda não está comprovada, constituindo apenas uma hipótese.

Rohde (2003) revela uma contribuição importante de fatores genéticos nas causas do TDAH - crianças com pais portadores do transtorno têm duas a oito vezes mais chance de adquirir a doença. Segundo Cypel (2007), os estudos de Safer (1973) apresentaram que irmãos têm mais incidências de ter TDAH do que meio irmãos. Entretanto, em virtude do pequeno número de estudos, não se permite indicar que a causa do TDAH seja hereditária.

Observaçôes permitem inferir que mesmo havendo possibilidade de participação de um fator genético, é muito provável que outros fatores familiares e ambientais intervenham na determinação do TDAH .(CYPEL, 2007)

Fatores ambientais como desentendimento familiar e presença de transtornos mentais dos pais, classe social baixa, família muito numerosa, criminalidade dos pais e colocação em lar adotivo, têm associação positiva com TDAH. Outra associação importante é com mães que apresentaram complicaçôes na gestação (eclampsia, duração prolongada do parto, má saúde materna) e adversidades durante a gravidez (uso de álcool, nicotina e drogas em geral). (ROHDE, 2003)

Importante ressaltar que a maioria dos estudos sobre pos-
síveis agentes ambientais apenas evidencia uma associaçáo
desses fatores com o TDAH, não sendo possível estabele-
cer uma relaçáo clara de causa e efeito entre eles (FARAO-
NE e BIEDERMAN apud ROHDE, 2003)

Benczik (2000) aponta, ainda, outros fatores que poderiam causar o TDAH: lesão cerebral, fatores neuroquímicos, fatores neurofisiológicos, chumbo, substâncias ingeridas durante a gravidez, fatores psicossociais e estressantes, como alto grau de discórdia conjugal, baixa instrução da mãe, família com apenas um dos pais, famílias com nível socioeconômico mais baixo.

\section{Avaliação diagnóstica do TDAH}

O diagnóstico do TDAH tem que ser preciso, pois não existe nenhum teste físico, neurológico ou psicológico que possa provar sua existência. Exames de sangue, urina, ressonância magnética ou tomografia computadorizada não resolverão o problema, nem, tampouco, farão o diagnóstico. A avaliação mais freqüente e que traz mais resultados é a feita através de entrevista com a criança, com seus pais e através de informaçôes da escola. Essa entrevista dura algumas horas e como, muitas vezes, a criança se mostra tranqüila e quieta no consultório, por se tratar de uma situação não rotineira, pode levar o avaliador ao erro. (PHELAN, 2005)

De acordo com Rohde (2003) o diagnóstico do TDAH como é fundamentalmente clínico, deve ter por base critérios claros e bem definidos. Esse diagnóstico é estabelecido por critérios internacionais de doenças, utilizando o CID10 e o DSM-IV.

O CID é um manual de classificação internacional de doenças e o DSM-IV - Diagnostic and Statistical Manual of Mental Disorders - Fourth Edition, é a $4^{\text {a }}$ edição do Manual Diagnóstico e Estatístico de Transtornos Mentais da Associação Pediátrica Americana, principal referência de diagnóstico para os profissionais de saúde mental dos Estados Unidos da América sendo também utilizado no Brasil. A Tabela I apresenta um quadro com resumo do DSM-IV no qual são elencados critérios diagnósticos. 
Tabela I - Quadro com resumo da 4a edição do Manual Diagnóstico e Estatístico de Transtornos Mentais da Associação Pediátrica Americana

A criança tem que se enquadrar em seis ou mais dos nove itens de uma ou ambas as listas de sintomas DSM-IV para desatenção ou hiperatividade/impulsividade.

\section{Desatenção:}

a) Não consegue prestar atenção em detalhes e comete erros por descuido;

b) Tem dificuldade de manter a atenção na tarefa ou na brincadeira;

c) Não ouve quando alguém lhe dirige diretamente a palavra;

d) Não consegue terminar as tarefas escolares, os afazeres domésticos ou os deveres do trabalho;

e) Tem dificuldade em organizar atividades;

f) Evita atividades que requerem esforço mental prolongado;

g) Perde coisas;

h) Distrai-se facilmente;

i) É esquecida

Hiperatividade:

a) Fica irrequieta ou se contorce na cadeira;

b) Sai do lugar quando se espera que permaneça sentada;

c) Corre de um lado para o outro ou escala coisas em situaçôes em que isso é inadequado;

d) Tem dificuldade de brincar em silencio;

e) Age como se fosse "movido a pilha";

f) Fala em excesso;

Impulsividade:

g) Responde antes que a pergunta seja completada;

h) Tem dificuldade de esperar sua vez;

i) Interrompe os outros ou se intromete.

Fonte: Phelan (2005)

\section{Principais comorbidades}

Comorbidade é um termo utilizado para caracterizar a ocorrência de dois ou mais transtornos em um mesmo indivíduo. Segundo Pereira et al (2005), a presença de comorbidades com o TDAH é comum em 30\% a 50\% dos casos.

As principais comorbidades com TDAH, segundo Rohde (2003), são:
- Transtorno Desafiador de Oposição (TDO), onde a criança apresenta um comportamento desafiador, opositor e implicante em relação a figuras de autoridade, em especial aos pais e professores, além de ter um humor facilmente irritável. Está presente em 35\% a 65\% dos pacientes com TDAH.

- Transtornos de Conduta (TC), que apesar de uma freqüência menor que o TDO, estão estimados de $20 \%$ a 50\% dos casos de TDAH. A principal característica é o desrespeito aos direitos básicos dos outros, como a integridade física e a propriedade. São exemplos de atitudes de pacientes com TC, mentiras freqüentes, fugas de casa, crueldade com animais e pessoas.

- Depressão, que se expressa por humor triste ou irritável, perda de interesse por atividades que normalmente são prazerosas, alteração de apetite, fadiga fácil, culpa excessiva e, eventualmente, idéias de suicídio. Ocorrem em 15\% a 75\% dos casos. Segundo Rohde (2003) alguns autores como Gittelman (1985) e Mannuzza (1993) não concordam com estes índices e em estudo realizado na Universidade Federal do Rio de Janeiro (UFRJ) por Souza e cols.(2001) foi encontrada uma prevalência de aproximadamente 14\%.

- Transtorno do Humor Bipolar, que em adultos é caracterizado por humor exaltado, euforia, idéias de grandiosidade e agitação psicomotora. As crianças apresentam sintomas mistos, aumento da irritabilidade e da agressividade, com episódios de explosão de agressividade. O diagnóstico em crianças é difícil, pois os sintomas podem ser confundidos com outros transtornos. A comorbidade com TDAH aumenta a inadequação social, o risco de suicídio e altera o prognóstico.

- Transtorno de Ansiedade - crianças com este transtorno demonstram medos ou preocupaçóes excessivas que interferem da adaptação acadêmica, social e familiar. Situaçôes corriqueiras geram extrema ansiedade e sofrimentos, eventos futuros também são fontes de preocupação. Segundo Rodhe (2003) nos estudos de Biederman e cols (1991 e 1999) a prevalência de Transtorno de Ansiedade com TDAH foi de 30\% a 40\%.

- Transtorno de Tiques (TT) - os tiques são movimentos ou vocalizaçôes repentinos, rápidos, recorrentes, não-rítmicos, estereotipados. O TT pode ser dividido em transtorno de tourette (acarreta a existência de diversos tiques motores e pelo menos um tique vocal), tique motor crônico, tique vocal crônico e transtorno de tique transitório. Estima-se que $60 \%$ das crianças com Transtorno de Tourette tenham também TDAH. (RODHE, 2003) 
Pereira et al, (2005) apresenta também como uma das principais comorbidades o Distúrbio do Desenvolvimento da Coordenação (DDC) que pode chegar a 50\% dos casos. Crianças com DDC têm dificuldades com habilidades motoras que requerem coordenação, além de apresentarem problemas acadêmicos e de sociabilidade. O diagnóstico é feito através de cuidadoso exame motor associado à positividade de critérios de TDAH.

Para as crianças com TDAH, uma avaliação sistemática de comorbidades auxilia a correta orientação da família quanto aos problemas que podem, inclusive, ser mais prejudiciais que o próprio TDAH ou, somar-se a ele, agravando o prognóstico final (PEREIRA et al, 2005).

\section{Desempenho escolar e o TDAH}

Quando o TDAH está presente pode causar problemas para a vida da criança em todas as esferas - em casa, em eventos sociais e principalmente afeta seu desenvolvimento na escola.

Segundo Rohde (2003), acontece um grande impacto no desenvolvimento educacional de crianças com TDAH. A desatenção e a falta do autocontrole, características do transtorno, intensificam-se em situaçóes de grupo, dificultando, ainda mais, a percepção dos estímulos relevantes, a estruturação a execução adequada das tarefas.

Estudos de Mannuzza et al (apud Pastura, 2005), observaram que portadores de TDAH tinham 2,5 anos de estudo a menos, comparado a jovens normais da mesma idade; além disso, 23\% destes não completaram seus estudos. Somente $12 \%$ dos portadores da doença conseguiram o diploma de nível superior. Já Rohde (apud Pastura, 2005) observou em escolares de Porto Alegre, que $87 \%$ dos portadores de TDAH possuíam mais de uma repetência em seus currículos, comparado a $30 \%$ dos não portadores.

A maioria das crianças com TDAH deseja ter um bom rendimento escolar como as demais crianças; no entanto, a dificuldade de concentração e motivação, aliada a uma estrutura escolar inadequada para esses alunos, dificultam o seu desempenho, levando-os normalmente a conflitos com professores e colegas de turma. Na população em geral, de $10 \%$ a $15 \%$ das crianças apresentam dificuldades de aprendizagem; em portadores de TDAH este número sobe para próximo de 40\%. (PHELAN, 2005)

\section{CONCLUSÃO}

É importante ressaltar que o Transtorno de Déficit de Atençâo e Hiperatividade (TDAH) é uma doença reconhe- cida pela OMS, cuja prevalência mundial gira em torno de $5 \%$ e ocorre tanto em meninos quanto em meninas. Pode ser considerada alta a comorbidade com outros transtornos, chegando a ocorrer em 30\% a 50\% dos casos. Ainda não existem certezas sobre as causas do TDAH, mas existem algumas hipóteses como fatores hereditários, fatores neuroquímicos e psicossociais que, quando associados, adquirem maior força na determinação do quadro. O diagnóstico ainda é puramente clínico e quanto mais cedo for detectado, melhor será o seu tratamento.

Pode ser verificado que é freqüente o baixo desempenho escolar nesses alunos, chegando a $40 \%$ dos portadores do TDAH apresentando dificuldades nas atividades acadêmicas. Tais dificuldades, por sua vez, mantêm o quadro e muitas vezes o agravam, uma vez que o aluno pode ser cada vez mais marginalizado. Resulta daí mais um dos motivos fundamentais para que o profissional da educação venha a conhecer o TDAH e possa encaminhar seus portadores para o tratamento adequado.

$O$ tratamento desses pacientes engloba uma equipe multidisciplinar, e o envolvimento da família é fundamental, por isso, é importante que além de médicos e psicólogos, que atuam com freqüência com transtornos psíquicos, pedagogos, professores e pais tenham mais informaçóes sobre o TDAH. Quanto maior os conhecimentos sobre o transtorno, melhores poderão ser as estratégias para minimizar seus efeitos negativos.

\section{REFERÊNCIAS BIBLIOGRÁFICAS}

BARBOSA, A. G. A.; BARBOSA G. A. ; AMORIM G. G. Hiperatividade conhecendo sua realidade. São Paulo. Casa do Psicólogo, 2005. 124 p.

BENCZIK, E. B. P. Transtorno de déficit de atenção/hiperatividade: atualizaçáo diagnóstica e terapêutica: um guia de orientaçáo para profissionais. São Paulo. Casa do Psicólogo, 2000. 110 p.

CAETANO, D.; CID-10 Classificaçáo de Transtornos Mentais e de Comportamento. Porto Alegre. Artmed, 1993, $351 \mathrm{p}$.

CIASCA, S.M. Distúrbios de aprendizagem: proposta de avaliaçáo interdisciplinar. São Paulo. Casa do Psicólogo, 2003. 220 p.

CYPEL, S. Défict de Atençáo e Hiperatividade e as Funçóes Executivas. $3^{\circ}$ ed. São Paulo. Lemos Editorial, 2007. $135 \mathrm{p}$. 
FONTANA, R.S. et al. Prevalência de TDAH em quatro escolas públicas brasileiras. Arq Neuropsiquiatr. 65(1): 134 $-137,2007$.

GOMES, M. et al. Conhecimento sobre Transtorno do Déficit de Atenção Hiperatividade no Brasil. J Bras Psiquiatr . 56(2): $94-101,2007$.

JORGE, M. R.; Manual Diagnóstico e Estatistico de Transtornos Mentais - DSM-IV-TR. 4o Ed. Porto Alegre. Artmed, 2002, 880 p.

PASTURA, M.C.G et al. Desempenho Escolar e Transtorno do Déficit de Atençáo e Hiperatividade. Ver. Psiq. Clín. 32(6): 324 - 329, 2005.

PEREIRA, H.S. et al. Transtorno do déficit de atenção e hiperatividade (TDAH): aspectos relacionados à comorbidade com distúrbios da atividade motora. Rev. Bras. Saúde Matern. Infant. 5(4): 391- 402, out./dez, 2005.

PHELAN, T. W. TDA/TDAH - Transtorno de Déficit de Atenção e Hiperatividade. São Paulo. M. Books do Brasil Editora Ltda, 2005, 246 p.

ROHDE, L. A. e cols. Princípios e Práticas em Transtorno de Déficit de Atenção e Hiperatividade. Porto Alegre. Artmed, 2003, 236 p. 